\title{
Relation between axial length of the eye and hypotensive effect of latanoprost in primary open angle glaucoma
}

\author{
E Arranz-Marquez, M A Teus
}

Br J Ophthalmol 2004;88:635-637. doi: 10.1136/bjo.2002.010827

Aims: To study the effect of axial length on the hypotensive effect of latanoprost in primary open angle glaucoma (POAG) in a prospective, observational study.

Methods: The authors measured axial length and baseline intraocular pressure (IOP) of 109 eyes with POAG, and then repeated the IOP measurements at 1, 3, and 6 months after starting treatment with latanoprost.

Results: The mean IOP level was significantly lower in eyes with a shorter axial length compared with the eyes with a longer axial length both at 3 and 6 months of treatment $(p=0.03$ and $p=0.04$, respectively, ANOVA).

Conclusion: The hypotensive effect resulting from treatment with latanoprost could be related to ocular axial length.

I atanoprost has proven to be a potent ocular hypotensive agent $^{1}$; nevertheless, some authors have observed a -heterogenic response to latanoprost. ${ }^{2}$ Therefore, we thought it would be interesting to identify the particular ocular characteristics that may be responsible for the variability in the response to latanoprost.

Aung et al reported recently that latanoprost is more effective than timolol in patients with chronic angle closure glaucoma (CACG). ${ }^{3}$ The reason for this high efficacy of latanoprost in CACG is unclear; the changes that occur in the angle morphology or the inflammation that may follow angle closure may facilitate the effect of latanoprost in these eyes.

Angle closure glaucoma occurs more commonly in eyes that have an anatomic predisposition (narrow angles), ${ }^{4}$ and it is more common in patients with hyperopia. For this reason, we analysed the relation between the hypotensive effect of latanoprost and the ocular axial length in primary open angle glaucoma (POAG), thus avoiding the confounding effect of peripheral angle synechiae.

\section{PATIENTS AND METHODS}

We studied prospectively 109 consecutive patients who met all the following inclusion criteria: diagnosis of POAG; normal anterior segment; no history of previous ocular surgery; episodes of angle closure glaucoma, or argon laser trabeculoplasty. Patients with previous laser iridotomy were included if the procedure had been performed at least 3 months before entrance into the study.

Both newly diagnosed patients and those who had been treated previously with antiglaucoma therapy that was considered ineffective or was not well tolerated were also included. No patient had been treated with any prostaglandin analogue before entering the study. When both eyes of the same patient met the inclusion criteria, the eye with the higher intraocular pressure (IOP) was selected; if both eyes had the same IOP, one eye was randomly selected.

Biometry was performed on the first visit. We used the automatic stop mode on the ultrasound device to take eight measurements of each eye, and the average was calculated. The eyes were classified into three groups according to the axial length. Eyes with an axial length of less than $22 \mathrm{~mm}$ were classified as short axial length (SAL); if the axial length was $22-25 \mathrm{~mm}$, the eyes were classified as medium axial length (MAL); and if axial length was greater than $25 \mathrm{~mm}$, the eyes were classified as having a long axial length (LAL). ${ }^{5}$

The baseline IOP was measured on the first visit. If the patients were being treated for glaucoma, a 3 week washout period was required before the basal IOP was determined.

Treatment was started with latanoprost $0.005 \%$ once daily. Applanation tonometry was performed at 1, 3, and 6 months after the start of treatment. Two consecutive readings were taken of each eye, and the average was recorded.

We defined a hyporesponder as a patient who had a reduction in IOP of less than $10 \%$ after 3 months of treatment; a hyperresponder was defined as a patient who had a response of $40 \%$ or more.

\section{RESULTS}

One eye from each of 109 patients ( 52 women, 52 men) were included in the study. Fourteen eyes had had YAG laser iridotomies for potential occlusion of narrow angles.

The mean axial length was $23.2 \mathrm{~mm}$ (range 19.3$32.7 \mathrm{~mm}$ ). Seventy eyes had medium axial lengths, 21 long axial lengths, and 18 short axial lengths. The mean (standard error of mean, SEM) anterior chamber depth was 2.66 (0.32) $\mathrm{mm}$.

The mean baseline IOP was 24.5 (4) mm Hg. There were no statistically significant differences between the axial length groups ( $p=0.8$ by ANOVA). One month after treatment, the IOP decreased significantly in the three study groups. The mean IOP was 17.7 (3) $\mathrm{mm} \mathrm{Hg}$ in the MAL group, 18.2 (3) $\mathrm{mm} \mathrm{Hg}$ in the LAL group, and 18.7 (4) $\mathrm{mm} \mathrm{Hg}$ in the group with a SAL. There were no statistically significant differences between the groups ( $p=0.5$ by ANOVA) (fig 1 ).

Three months after treatment was started, the mean (SEM) IOP was 17.4 (3.5) $\mathrm{mm} \mathrm{Hg,} 18.2$ (4.1) $\mathrm{mm} \mathrm{Hg}$, and 15.8 (3.5) $\mathrm{mm} \mathrm{Hg}$, respectively, in the groups with medium, long, and short axial lengths. There was a statistically significantly greater decrease in IOP in the group with a SAL than the group with a LAL ( $p=0.04$, ANOVA) (fig l).

After 6 months of therapy with latanoprost, the mean (SEM) IOP levels were 17.2 (3.7) $\mathrm{mm} \mathrm{Hg,} 17.8$ (3.2) $\mathrm{mm} \mathrm{Hg}$, and 15.5 (2.8) $\mathrm{mm} \mathrm{Hg}$, respectively, in the three groups. There was a statistically significantly greater reduction in IOP in the group with a SAL compared with the group with a LAL ( $p=0.03$ by ANOVA) (fig 1$)$.

When we compared the groups of short and long axial length, and classified the patients as hyperresponders or

Abbreviations: $C A C G$, chronic angle closure glaucoma; IOP, intraocular pressure; LAL, long axial length; MAL, medium axial length; $M M P$, matrix metalloproteinase; POAG, primary open angle glaucoma; SAL, short axial length. 


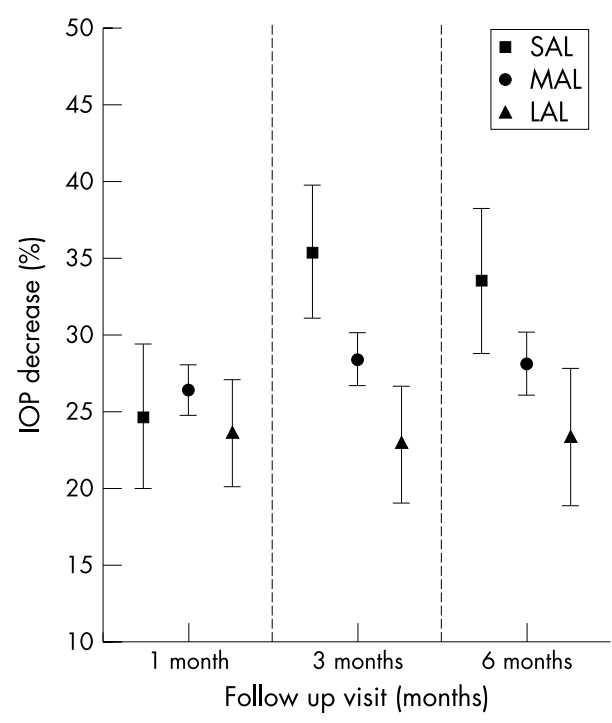

Figure 1 Mean (SEM) decrease in IOP values (\%) in the groups with long (LAL), medium (MAL), and short (SAL) axial lengths at 1,3 , and 6 months of latanoprost treatment.

hyporesponders to latanoprost, we found that most eyes that had a minimal response to latanoprost had LAL, whereas most of the eyes that had a greater than normal response had SAL ( $p=0.04, \chi^{2}$ test) (fig 2 ).

An evaluation of the anterior chamber depth in the hyperresponders and the hyporesponders did not show a statistically significant difference.

Nineteen patients were lost to follow up after 3 months, 17 of them because of poor IOP control (one in the group with SAL, four in the group with LAL, and 12 in the group with $M A L)$, and the other two because of poor tolerance.

\section{DISCUSSION}

We found that eyes with POAG and an SAL have a better response to treatment with latanoprost than eyes with an LAL. To our knowledge, this has not been previously reported. The mechanism of the relation between axial length and the response to latanoprost is unknown.

It is generally accepted that latanoprost decreases IOP by increasing the uveoscleral outflow of the aqueous humour,

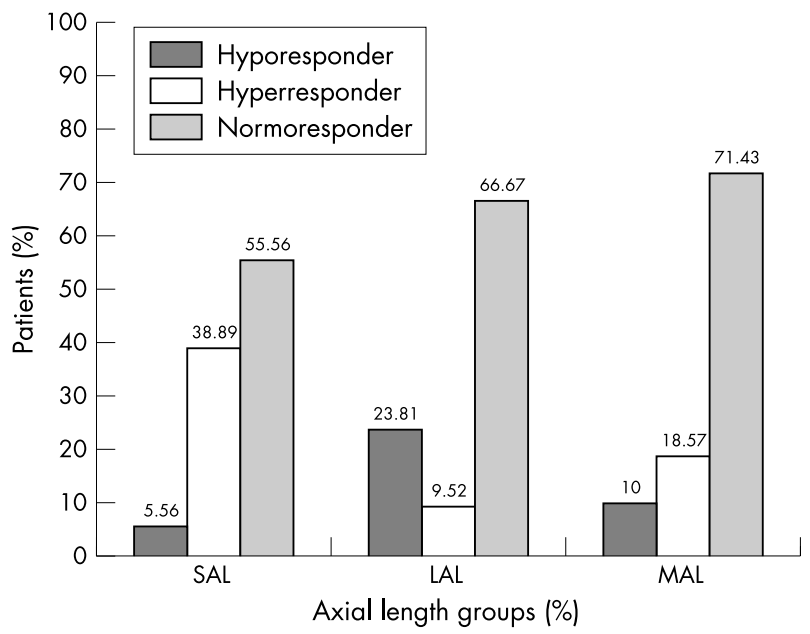

Figure 2 Distribution (\%) of hyporesponders, hyperresponders, and normoresponders eyes in the groups with short (SAL), medium (MAL), and long (LAL) axial lengths. that finally leaves the eye through the sclera (transscleral outflow). ${ }^{6}$

Eyes with a longer axial length usually have thinner sclera, and eyes with a shorter axial length tend to have thicker sclera. ${ }^{7}$ It is widely accepted that nanophthalmic eyes have a reduced transscleral flow that results from a thick sclera that decreases the uveoscleral outflow. ${ }^{8}$

A number of studies have pointed to the possible role of matrix metalloproteinases (MMPs) in the pathogenesis of increased axial length in myopia. ${ }^{9}$ These enzymes also seem to be involved in the mechanism of action of latanoprost. ${ }^{10}$

Aung and colleagues reported the superior IOP lowering effect of latanoprost compared with timolol in $C A C G,{ }^{3}$ but the mechanism was not clear; in fact, it has been shown recently that its efficacy is independent of the height of the ciliary face. ${ }^{11}$ According to our results, it may be that it is the short axial length of these eyes, and not the angle closure, that makes them more sensitive to the ocular hypotensive effect of latanoprost.

Because eyes with shorter axial lengths have a thicker sclera, a diminished basal uveoscleral outflow possibly may result. Therefore, the potential hypotensive effect of latanoprost could be greater, because this aqueous outflow pathway is underused. Another possibility is that if the MMPs are active in the growth of the globe that occurs while myopia is developing, then these eyes may become less sensitive to these enzymes, which in turn seem to be the ones that regulate the effect of latanoprost at the biochemical level.

The difference in IOP reduction between the SAL and LAL groups was not apparent until 3 months after latanoprost therapy started, and this may be related to the fact that some time may be needed for the MMPs to degrade the extracellular matrix and increase uveoescleral outflow.

Given the greater efficacy of latanoprost in lowering IOP in eyes with a short axial length, this agent could be considered as the first line therapy in these eyes with either CACG or POAG. It is clear that more studies are needed to further elucidate the factors that affect the variability in hypotensive response to prostaglandin analogues.

\section{Authors' affiliations \\ E Arranz-Marquez, M A Teus, University of Alcalá, Hospital Oftalmológico Internacional de Madrid}

Correspondence to: Dr E Arranz-Marquez, Chiquinquira 56, Madrid, Spain; esther-arranz@ole.com

Accepted 30 April 2003

\section{REFERENCES}

1 Camras CB, The United States Latanoprost Study Group. Comparison of latanoprost and timolol in patients with ocular hypertension and glaucoma: a six month, masked, multicenter trial in the United States. Ophthalmology 1996; 103:138-47.

2 Patelska B, Greenfield DS, Liebmann JM. Latanoprost for uncontrolled glaucoma in a compassionate case protocol. Am J Ophthalmol 1997; 124:279-86.

3 Aung T, Wong HT, Yip CC. Comparison of the intraocular pressure-lowering effect of latanoprost and timolol in patients with chronic angle closure glaucoma. Ophthalmology 2000;107:1178-83.

4 Gohdo T, Tsumura T, lijima H, et al. Ultrasound biomicroscopic study of ciliary body thickness in eyes with narrow angles. Am J Ophthalmol 2000; 129:342-6.

5 Ruiz M, Mendicute J. Medida de la longitud axial. In: Mendicute J, ed. Biometría, fórmulas y manejo de la sorpresa refractiva en la cirugía de catarata. Monografías de la Sociedad Española de Cirugía Ocular ImplantoRefractiva. Tecnimedia Editorial, 2000:38-40.

6 Toris CB, Camras CB, Yablonski ME, et al. Effects of exogenous prostaglandins on aqueous humor dynamics and blood-aqueous barrier function. Surv Ophthalmol 1997;41:S69-S75.

7 McBrien NA, Cornell LM, Gentle A. Structural and ultrastructural changes to the sclera in a mammalian model of high myopia. Invest Ophthalmol Vis Sci 2001;42:2179-87. 
8 Gass JDM. Uveal effusion syndrome: A new hypothesis concerning pathogenesis and technique of surgical treatment. Trans Am Ophthalmol Soc 1983;71:246-60.

9 Rada JA, Perry CA, Slover ML, et al. Gelatinase A and TIMP-2 expression in the fibrous sclera of myopic and recovering chick eyes. Invest Ophthalmol Vis Sci 1999;40:3091-9.
10 Weinreb RN, Kashiwagi K, Kashiwagi F, et al. Prostaglandins increase matrix metalloproteinase release from human ciliary smooth muscle cells. Invest Ophthalmol Vis Sci 1997;38:2772-80.

11 Ritch R, Ishikawa H, Rothman R, et al. The efficacy of latanoprost is independent of the width of the ciliary body face. J Glaucoma 2002;11:239-43. 